Interactive comment on "Landslide susceptibility assessment based on different machine-learning methods in Zhaoping County of eastern Guangxi" by Chunfang Kong et al.

Chunfang Kong et al.

xukai@cug.edu.cn

Received and published: 17 October 2020 


\section{Response to SC1 from Zhu Liang}

October 17, 2020

\section{Dear Zhu Liang:}

Thank you for your comments concerning our manuscript ID nhess-2020-251 (Landslide susceptibility assessment based on different machine-learning methods in Zhaoping County of eastern Guangxi). Those comments are all valuable and very helpful for revising and improving our paper, as well as of important guiding significance to our researches. We have studied comments carefully and have made correction which we hope meet the suggestions. Revised portion are marked in highlight in the paper. The main corrections in the paper and the responds to the reviewer's comments are as flowing:

1. What is your research question? Can you really claim in 2020 that the aim of the research was to compare algorithms and their respective performance? How many articles are out there with the exact same question and structure and plots? This field of geomorphology has become an empty shell with no research question whatsoever other than let's measure the delta AUC and let's see how many decimal places down the line, we can claim a model to be better than the other.

Printer-friendly version

Discussion paper 
The geological environment in eastern Guangxi is fragile and geological hazards occur frequently, which not only causes huge economic losses and ecological environment damage, but also seriously restricts the survival of human beings and the sustainable development of human society (Pourghasemi et al., 2012; Uitto and Shaw, 2016). In particular, with the rapid development of the economy in recent decades, the frequency and intensity of the geological hazards are rapidly increasing with the over-exploitation and utilization of natural resources by humans (Guzzetti et al., 1999). Therefore, it is of great significance to objectively evaluate the susceptibility of geological hazards for the reduction and prevention of the disasters. Please see L32-39.

The objective of the present paper is to seek a method to quickly and accurately evaluate the susceptibility grade of landslide for Zhaoping County of Guangxi. To this end, a total of ten factors of high correlation with landslide disaster occurrence were selected for running four machine-learning (ML) methods, and the landslide susceptibility grade was quickly and accurately evaluated by model simulation in Zhaoping County, providing methodological support for engineering construction, ecological environment construction, rapid economic development, and disaster reduction and disaster prevention for Guangxi.

2. There are hundreds of articles published every year on model comparison. They are all equally vague and they equally do not provide any practical solution to a real problem. To prove this statement to you, I would suggest you to search on Scopus using the following keywords: "Landslide susceptibility", "comparison" (and possibly "ensemble"). All the articles will have the same structure, similar results and similar conclusions as those in the present manuscript. To me, this looks more like a technical report rather than a scientific contribution of relevance, sorry.

Indeed, I admit that hundreds of articles published every year on model comparison, and each researcher wants to explore a common model for rapid and

Printer-friendly version Discussion paper

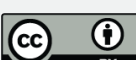


accurate evaluation the susceptibility grade for landslide disasters. However, this work is more complex and requires long-term efforts by all researchers.

At the same time, the occurrence of landslide disasters is very different in different regions and different geological environment, so, a large number of landslide models are produced for different regions. This paper aims at the ecological environment characteristics of landslide disaster in Zhaoping County, hoping to find a suitable model to quickly and accurately evaluate the susceptibility grade for landslide disaster in Guangxi.

\section{The sampling strategy. There is obvious non-parallel data between land- slide point and non-landslide point (1:6). How to avoid machine learning preference?}

Field investigation showed that there were 345 landslide disaster sites in the study area, all of which participated in the training and testing of the model. Among them, $242(70 \%)$ landslide hazards points were selected as training set samples, and $103(30 \%)$ landslide disaster points were selected as testing set samples. For non-disaster points, training and testing datasets were constructed by random sampling method based on environmental similarity. Among them, 1,251 non-hazards points with low environmental similarity with landslide disaster points were selected as training set samples, and 939 non-hazards points with low environmental similarity with landslide disaster points were selected as testing set samples. Therefore, in view of the obvious non-parallel data between landslide points and non-slide points in the study area, random sampling method based on environmental similarity strategies was adopted to construct training set and testing set to avoid machine learning preference. Please see the L181-182 and L183-184.

In summary, we are very grateful for your comments, and those comments are all valuable and very helpful for revising and improving our paper, as well as of important

Printer-friendly version

Discussion paper 
guiding significance to our researches.

NHESSD

Interactive

comment 